\title{
Digital Hologram Compression Using Correlation of Reconstructed Object Images
}

\author{
Jae-Young Sim \\ School of Electrical and Computer Engineering, \\ Ulsan National Institute of Science and Technology, \\ Ulsan 689-798, South Korea \\ jysim@unist.ac.kr
}

\begin{abstract}
An efficient digital hologram compression algorithm is proposed using the correlation in the complex valued object image. While the pure values are almost uncorrelated, the magnitude values exhibit a strong correlation between the real and imaginary part object images. Therefore, we adaptively employ the encoding result of one image to encode another image. Both images are first wavelet transformed and the wavelet coefficients are encoded using the SPIHT method. We used the significance encoding result of the real part image as the contexts of arithmetic coder for encoding the imaginary part image. Experimental results demonstrate that the proposed algorithm yields a better compression performance than the conventional method.
\end{abstract}

Keywords: Digital hologram, digital hologram compression, contextadaptive arithmetic coding.

\section{Introduction}

Three-dimensional (3D) images facilitate more realistic and immersive visual experiences, and therefore have drawn much attention in recent years. Holography is considered as one of the most promising techniques for 3D image representation, since it is free from eye fatigue and viewpoint constraints. Fig. 11 shows a typical principle of holography [7]. A light wave is split into two parts such that one directly travels to the recording medium as a reference light, and the other illuminates a 3D object. The interference pattern between the reference light and the reflected light on the $3 \mathrm{D}$ object is recorded, which is called hologram. When the hologram is illuminated with the reference light, the virtual object image is reconstructed.

With the aid of high resolution image sensors and high performance computers, holograms can be stored and processed in digital form 813612. To be specific, an interference pattern is captured by a CCD (charged coupled device) sensor and stored as a digital image which is called digital hologram. In addition, the object image can be numerically calculated with computers based on the mathematical modeling of the reconstruction process 12 . The numerically reconstructed image yields the artifacts such as the bright region of undiffracted 


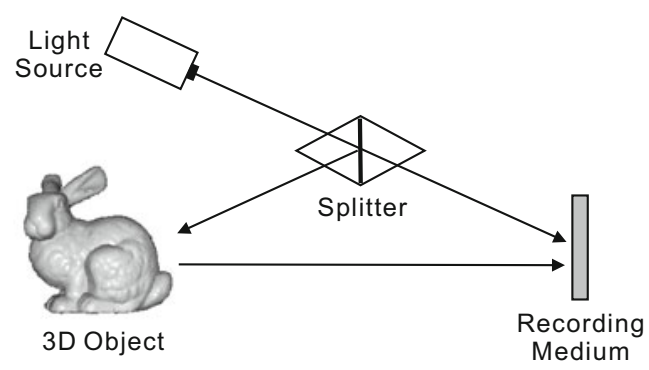

(a)

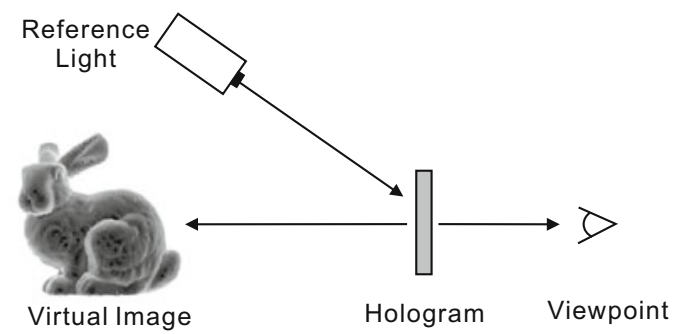

(b)

Fig. 1. Principle of holography. (a) Hologram recording and (b) image reconstruction

light wave and the conjugate object image. To remove such drawbacks, phaseshifting digital holography was developed which employs multiple interference patterns to directly extract the complex amplitude of the object wave [17.

In order to sufficiently sample the detailed shape of interference pattern, digital hologram is generally stored as a high resolution image which requires a huge amount of storage space. Therefore, a lot of research has been performed to efficiently compress the digital holograms 10 15 164453. Various entropy coding methods were used to losslessly compress the digital holograms [10]. Shortt et al. transformed the phase-shifting digital holograms using wavelets and encoded the quantized wavelet coefficients [15]. A nonuniform quantization method was also proposed based on the histogram of digital hologram [16]. While the most algorithms have directly compressed the digital hologram data, Darakis and Soraghan developed the compression algorithms for reconstructed images [45/3. The wavelet-like Fresnelet transform is used and the transformed coefficients of the reconstructed image are encoded [4. The compression performance of the digital hologram was also compared to that of the reconstructed image [5]3. Note that, progressive transmission can be facilitated in [5] and [3], since the embedded bitstreams are derived by using the SPIHT (set partitioning in hierarchical trees) method [11.

However, the previous compression techniques did not exploit the correlation in the complex valued reconstructed wave field from a digital hologram. In this paper, we investigate the correlation between the real and imaginary part data of 
the complex valued object image, and propose an efficient compression algorithm for digital holograms. It is observed that the magnitude images of the real and imaginary parts are highly dependent. Therefore we use the encoding result of the real part image as contexts to adaptively encode the imaginary part image based on the context-adaptive arithmetic coding. Experimental results demonstrate that the proposed algorithm exhibits a better rate-distortion performance than the existing method to compress digital holograms.

This paper is organized as follows. Section 2 describes the digital holography. The correlation of digital hologram is investigated in Section 3. Section 4 explains the coding algorithm of digital hologram data. Section 5] presents the experimental results, and finally Section 6 concludes this paper.

\section{Digital Holography}

Fig. 2 illustrates a coordinate system in digital holography, where the digital hologram is captured in the hologram plane (or camera plane) and the reconstructed image is calculated in the plane of distance $d$ from the hologram plane. Let $\mathcal{U}(x, y)$ be the complex amplitude of the reflected object wave in the hologram plane, which is represented by

$$
\mathcal{U}(x, y)=a_{u}(x, y) \exp \left\{i \phi_{u}(x, y)\right\}
$$

where $a_{u}(x, y)$ and $\phi_{u}(x, y)$ denote the real amplitude and the phase, respectively. Similarly, the reference light wave is given by

$$
\mathcal{V}(x, y)=a_{v}(x, y) \exp \left\{i \phi_{v}(x, y)\right\} \text {. }
$$

The intensity of the interference pattern of $\mathcal{U}(x, y)$ and $\mathcal{V}(x, y)$ is the scaled version of digital hologram, which is calculated as

$$
I(x, y)=|\mathcal{U}(x, y)+\mathcal{V}(x, y)|^{2}
$$

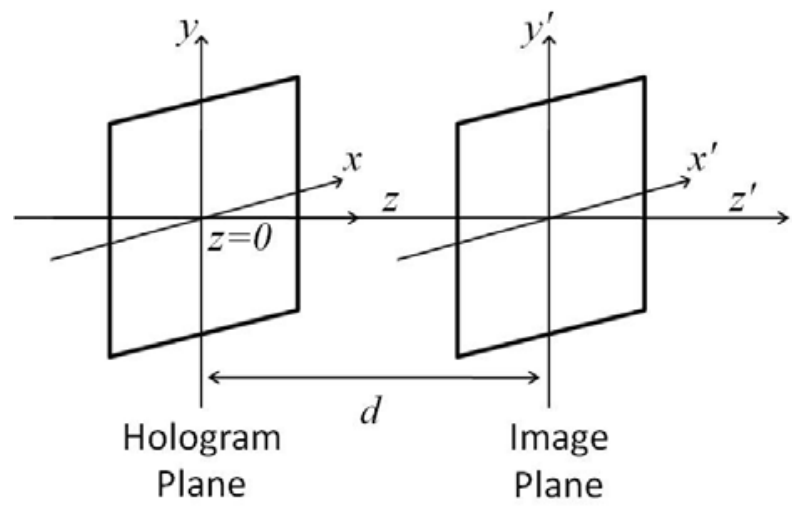

Fig. 2. Coordinate system in digital holography 


$$
\begin{aligned}
& =(\mathcal{U}(x, y)+\mathcal{V}(x, y))(\mathcal{U}(x, y)+\mathcal{V}(x, y))^{*} \\
& =a_{u}^{2}(x, y)+a_{v}^{2}(x, y)+\mathcal{U}(x, y) \mathcal{V}^{*}(x, y)+\mathcal{V}(x, y) \mathcal{U}^{*}(x, y)
\end{aligned}
$$

where $*$ means the complex conjugate.

When reconstructing an image from the digital hologram, $I(x, y)$ is multiplied by the reference wave as

$$
\mathcal{V}(x, y) I(x, y)=\left(a_{v}^{2}(x, y)+a_{u}^{2}(x, y)\right) \mathcal{V}(x, y)+a_{v}^{2}(x, y) \mathcal{U}(x, y)+\mathcal{V}^{2}(x, y) \mathcal{U}^{*}(x, y)
$$

The first term denotes the undiffracted component passing through the hologram. $a_{v}^{2}(x, y) \mathcal{U}(x, y)$ and $\mathcal{V}^{2}(x, y) \mathcal{U}^{*}(x, y)$ mean the virtual object image and the distorted real object image, respectively. In practice, as shown in Fig. 2, the complex amplitude $\mathcal{W}\left(x^{\prime}, y^{\prime}\right)$ in the image plane is reconstructed by propagating $\mathcal{V}(x, y) I(x, y)$ from the hologram plane to the image plane via the FresnelKirchhoff integral in (2), which models the diffraction of light wave [1412].

$$
\mathcal{W}\left(x^{\prime}, y^{\prime}\right)=\frac{i}{\lambda} \int_{-\infty}^{\infty} \int_{-\infty}^{\infty} \mathcal{V}(x, y) I(x, y) \frac{\exp \left(-i \frac{2 \pi}{\lambda} D\right)}{D} d x d y
$$

where $\lambda$ is the wavelength of the reference light and $D=\sqrt{\left(x-x^{\prime}\right)^{2}+\left(y-y^{\prime}\right)^{2}+d^{2}}$. Equation (2) is numerically approximated by

$$
\begin{aligned}
\mathcal{W}\left(x^{\prime}, y^{\prime}\right) & =\frac{i}{\lambda d} \exp \left(-i \frac{2 \pi d}{\lambda}\right) \exp \left\{-i \frac{\pi}{\lambda d}\left(x^{\prime 2}+y^{\prime 2}\right)\right\} \\
& \times \iint_{-\infty}^{\infty} \mathcal{V}(x, y) I(x, y) \exp \left\{\frac{-i \pi}{\lambda d}\left(x^{2}+y^{2}\right)\right\} \exp \left\{\frac{i 2 \pi}{\lambda d}\left(x x^{\prime}+y y^{\prime}\right)\right\} d x d y
\end{aligned}
$$

which is called Fresnel transform. Moreover, the digitized version of Fresnel transform can be efficiently calculated by using the fast Fourier transform [1412].

\section{Correlation in Digital Hologram}

The complex valued image $\mathcal{W}\left(x^{\prime}, y^{\prime}\right)$ of $3 \mathrm{D}$ object is reconstructed from a digital hologram using the diffraction of light waves via (2) or (3). Therefore, $\mathcal{W}\left(x^{\prime}, y^{\prime}\right)$ is composed of the real part image $\operatorname{Re}\left(\mathcal{W}\left(x^{\prime}, y^{\prime}\right)\right)$ and the imaginary part image $\operatorname{Im}\left(\mathcal{W}\left(x^{\prime}, y^{\prime}\right)\right)$

$$
\mathcal{W}\left(x^{\prime}, y^{\prime}\right)=\operatorname{Re}\left(\mathcal{W}\left(x^{\prime}, y^{\prime}\right)\right)+i \operatorname{Im}\left(\mathcal{W}\left(x^{\prime}, y^{\prime}\right)\right)
$$

Fig. 3 (a) represents the 'Brahms' hologram. Fig. 3 (b) and (c) show the magnitude images of the corresponding $\operatorname{Re}\left(\mathcal{W}\left(x^{\prime}, y^{\prime}\right)\right)$ and $\operatorname{Im}\left(\mathcal{W}\left(x^{\prime}, y^{\prime}\right)\right)$, respectively. While the digital hologram yields a noise-like pattern and thus does not directly represent the shape of captured object, the reconstructed image exhibits a relatively higher spatial correlation by showing the shape of object. Therefore, a more improved compression performance can be achieved on the reconstructed images than the digital holograms 5 53. Moreover, as observed in Fig. 3 (b) and 


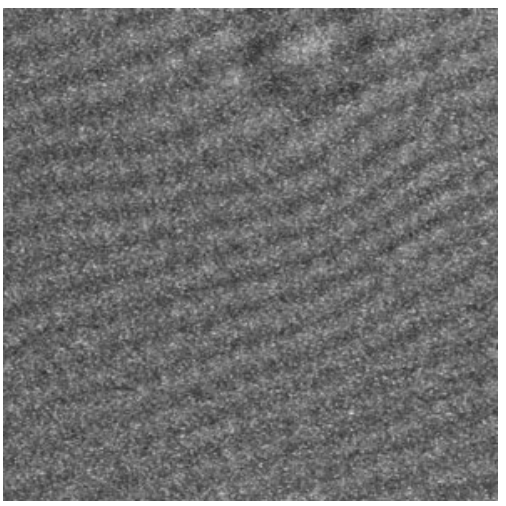

(a)

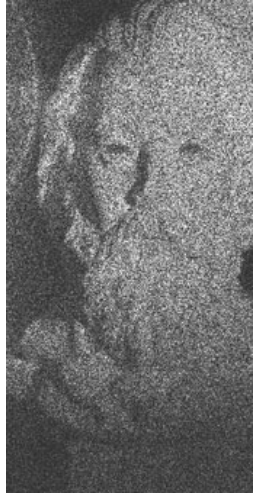

(b)

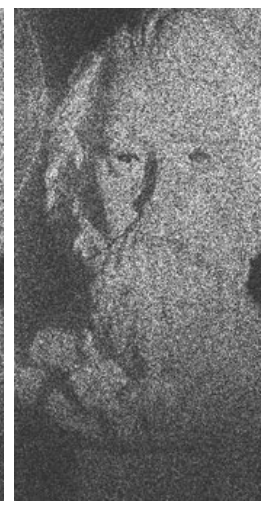

(c)

Fig. 3. Digital hologram and the reconstructed object images. (a) The 'Brahms' digital hologram. (b) Magnitude of the real part object image. (c) Magnitude of the imaginary part object image.

(c), the real and imaginary part images also yield the similar magnitude values each other, at the corresponding pixels.

In order to quantitatively investigate the dependence between $\operatorname{Re}\left(\mathcal{W}\left(x^{\prime}, y^{\prime}\right)\right)$ and $\operatorname{Im}\left(\mathcal{W}\left(x^{\prime}, y^{\prime}\right)\right)$, we measure the Pearson correlation coefficient

$$
\rho(R, I)=\frac{E[R-E[R]] E[I-E[I]]}{\sqrt{E\left[(R-E[R])^{2}\right] E\left[(I-E[I])^{2}\right]}}
$$

where $R$ and $I$ are the random variables of $\operatorname{Re}\left(\mathcal{W}\left(x^{\prime}, y^{\prime}\right)\right)$ and $\operatorname{Im}\left(\mathcal{W}\left(x^{\prime}, y^{\prime}\right)\right)$, respectively. In general, $\rho(R, I) \approx 0$. It means that the pure values of $\operatorname{Re}\left(\mathcal{W}\left(x^{\prime}, y^{\prime}\right)\right)$ and $\operatorname{Im}\left(\mathcal{W}\left(x^{\prime}, y^{\prime}\right)\right)$ are almost uncorrelated each other, yielding different patterns of speckles. However, it is empirically observed that the magnitude values of $\left|\operatorname{Re}\left(\mathcal{W}\left(x^{\prime}, y^{\prime}\right)\right)\right|$ and $\left|\operatorname{Im}\left(\mathcal{W}\left(x^{\prime}, y^{\prime}\right)\right)\right|$ have a relatively strong correlation, for example 0.6 in the 'Brahms' hologram.

The similar characteristics are also observed in the wavelet transformed images. Fig. 4(a) and (b) represent the magnitude images of the wavelet coefficients of $\operatorname{Re}\left(\mathcal{W}\left(x^{\prime}, y^{\prime}\right)\right)$ and $\operatorname{Im}\left(\mathcal{W}\left(x^{\prime}, y^{\prime}\right)\right)$, respectively, which are reconstructed from the 'Copper screw' hologram. The correlation coefficient for the wavelet coefficients is 0.53 .

\section{Digital Hologram Compression}

\subsection{Progressive Coding}

One of the state-of-the-art compression algorithms of digital holograms was developed on the reconstructed images using the wavelet-like transform and the SPIHT coding method [3. We also apply the wavelet transform to the real part and the imaginary part of the complex valued reconstructed image individually, 


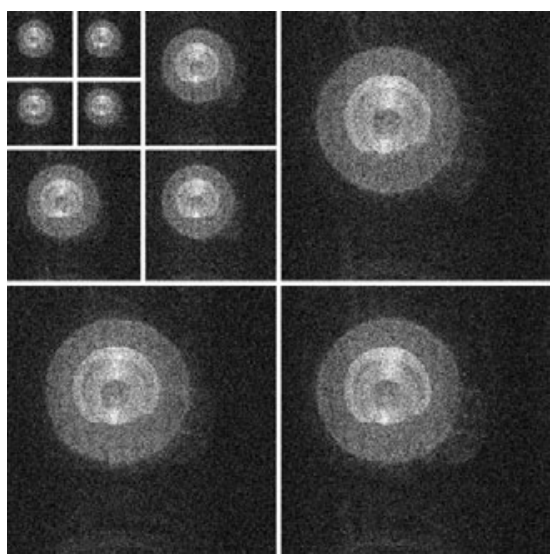

(a)

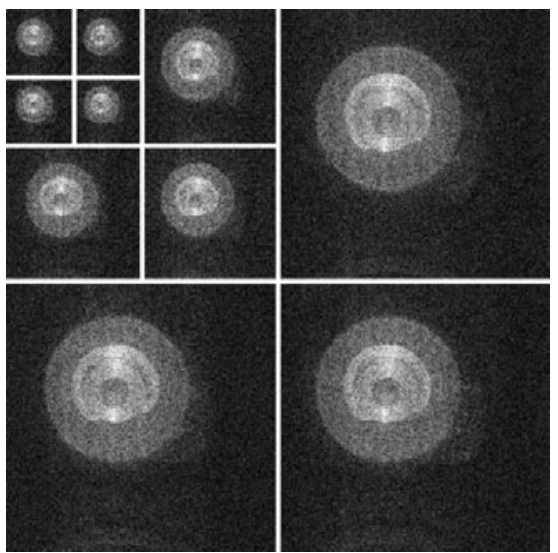

(b)

Fig. 4. Magnitude of wavelet coefficients in the object image reconstructed from the 'Copper screw' digital hologram. (a) Real part image. (b) Imaginary part image.

using the $9 / 7$ filters [2]. The wavelet coefficients are uniformly quantized with the same step size, and then progressively encoded using the SPIHT method [1]. As shown in Fig. 4, the reconstructed image of a digital hologram is relatively sparse in the sense of significant pixels when compared with the general images, since it represents the sectional image of 3D objects focused on a specific depth. As a result, a coding gain can be expected by encoding a group of pixels together in the reconstructed image.

\subsection{Context-Adaptive Coding}

For each image of the real and imaginary parts, bitplanes of the quantized wavelet coefficients are progressively encoded one by one from the most significant bitplane (MSB) to the least one based on the SPIHT method. Let $c(x, y)$ be the quantized wavelet coefficient at the pixel coordinate $(x, y)$. For a given $b$ th $\mathrm{MSB}$, the significance coding is performed. If $c(x, y)$ first becomes significant, i.e. $2^{b} \leq|c(x, y)|<2^{b+1}$, then the significance bit $S_{b}(c(x, y))=1$ is encoded and the sign bit is encoded. If $c(x, y)$ is insignificant as $|c(x, y)|<2^{b}$, then $S_{b}(c(x, y))=0$ is encoded. When $c(x, y)$ is already significant at the previous bitplane such that $|c(x, y)| \geq 2^{b+1}$, the magnitude refinement bit is encoded. The resulting bit sequence is entropy encoded using the arithmetic coder 9 .

The significance of wavelet coefficient is directly related to the magnitude which yields a strong correlation between the real and imaginary part images. It means that if the coefficient $c_{\text {real }}(x, y)$ in the real part image is significant (or insignificant) at a given bitplane, the coefficient $c_{\text {imag }}(x, y)$ in the imaginary part image is highly probable to be significant (or insignificant) at the same bitplane. Therefore, the result of significance coding for one image can be used to predict the significance of another image. In practice, $S_{b}\left(c_{r e a l}(x, y)\right)$ is first initialized to be ' 0 ' for all $b$, and then updated while encoding $c_{\text {real }}(x, y)$. When encoding the 
imaginary part image, if $S_{b}\left(c_{\text {real }}(x, y)\right)=1$, then $S_{b}\left(c_{\text {imag }}(x, y)\right)$ is arithmetic encoded with the context $A_{\text {pixel }}$, otherwise with the context $B_{\text {pixel }}$. Furthermore, we employ the different contexts according to the frequency subbands of wavelet decomposition, since the correlation coefficient between the real and imaginary part images exhibits different characteristics in each subband.

In addition, the SPIHT method improves the coding gain by encoding the significance of a group of coefficients together. Let $G(x, y)$ be the group of all pixel coefficients belonging to the hierarchical tree derived from the coordinate $(x, y)$. If all the coefficients in $G(x, y)$ are insignificant at the $b$ th MSB, then the significance bit $S_{b}(G(x, y))=0$ is encoded. Otherwise $S_{b}(G(x, y))=1$ is encoded, and the group $G(x, y)$ is divided into the smaller sub-groups. The significance coding is iteratively performed on each sub-group until the coordinates of significant coefficients are encoded. The group $G_{\text {real }}(x, y)$ in the real part image and the group $G_{i m a g}(x, y)$ in the imaginary part image are derived from the same spatial region, and thus yield the similar characteristics of significance. Therefore, we employ the other contexts $A_{\text {group }}$ and $B_{\text {group }}$ to adaptively encode $S_{b}\left(G_{\text {imag }}(x, y)\right)$, according to the result of $S_{b}\left(G_{r e a l}(x, y)\right)$ which is updated during the encoding of the real part image.

\section{Experimental Results}

The performance of the proposed algorithm is evaluated using the three digital holograms, 'Brahms', 'Dice', and 'Copper screw', whose reconstructed images are shown in Fig. 5. Table 1 provides the capturing conditions for these digital holograms. The distortion of the reconstructed image is measured by the normalized root mean square (NRMS) error [3], and given by

$$
\sqrt{\frac{\sum_{x} \sum_{y}\left(|\mathcal{W}(x, y)|^{2}-|\tilde{\mathcal{W}}(x, y)|^{2}\right)^{2}}{\sum_{x} \sum_{y}\left(|\mathcal{W}(x, y)|^{2}\right)^{2}}}
$$

where $\mathcal{W}(x, y)$ and $\tilde{\mathcal{W}}(x, y)$ denote the original image and the decoded one from the compressed bitstream, respectively.

Fig. 6. Fig. 7 and Fig. 8 show the rate and distortion compression performances for the imaginary part object images of the 'Brahms', 'Dice', and 'Copper screw' holograms, respectively. The dashed lines exhibit the results of the conventional coding method [3] and the solid lines represent the results of the proposed context-adaptive coding method. In order to compare the performances from only the coding schemes, we also used the $9 / 7$ filters [2] to get the wavelet coefficients in the conventional method. It is observed that the proposed compression algorithm provides a better rate-distortion performance compared with the conventional method which separately encodes the real and imaginary part images. The bitrate reduction is over $20 \%$ especially at low bitrates. For example, while the conventional method consumes 11417 bytes to encode the imaginary part image to provide the distortion $7.65 \times 10^{-2}$ for the 'Brahms' hologram, the proposed algorithm only requires 8863 bytes to achieve the same distortion. 


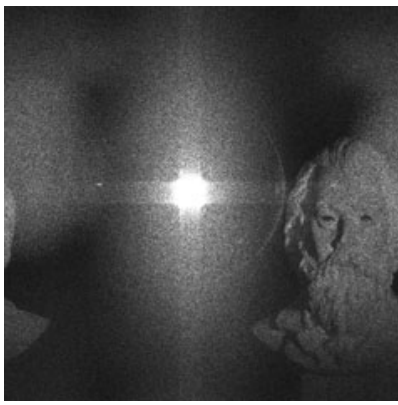

(a)

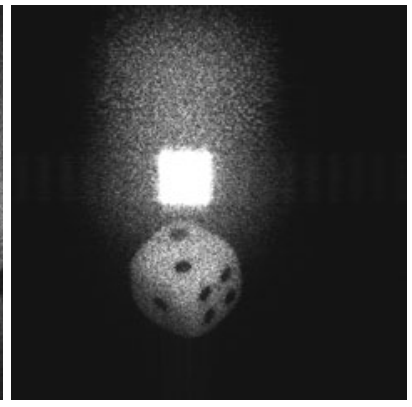

(b)

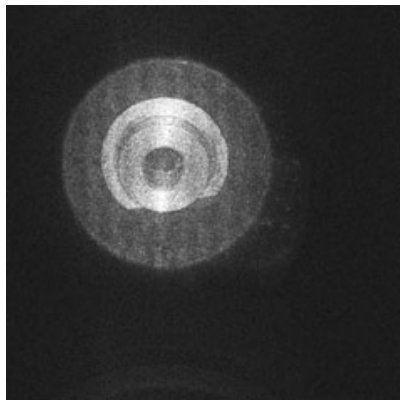

(c)

Fig. 5. Reconstructed images of the test digital holograms. (a) Brahms (Courtesy of the HoloVision project 1]). (b) Dice (Courtesy of Dr. U. Schnars). (c) Copper screw (Courtesy of Dr. F. Zhang). The 'Brahms' and the 'Dice' are the classic digital holograms and the 'Copper screw' is the phase-shifting digital hologram.

Table 1. Capturing conditions of digital holograms

\begin{tabular}{|c||c|c|c|}
\hline & Brahms & Dice & Copper screw \\
\hline \hline Type & classic & classic & phase shifting \\
\hline Image resolution & $1024 \times 1024$ & $1024 \times 1024$ & $1024 \times 1024$ \\
\hline Pixel size & $6.8 \mu \mathrm{m}$ & $6.8 \mu \mathrm{m}$ & $6.45 \mu \mathrm{m}$ \\
\hline Wavelengh of reference light & $632.8 \mathrm{~nm}$ & $632.8 \mathrm{~nm}$ & $830 \mathrm{~nm}$ \\
\hline Captured distance & $1290 \mathrm{~mm}$ & $1054 \mathrm{~mm}$ & $285 \mathrm{~mm}$ \\
\hline
\end{tabular}

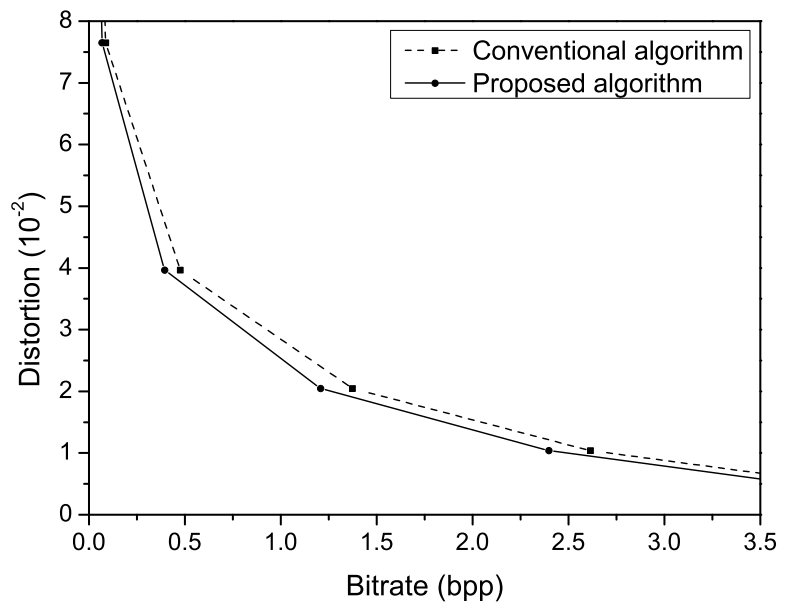

Fig. 6. Rate and distortion curve of the proposed algorithm compared with the conventional method. The rate means the average bits per pixel to encode the imaginary part object image reconstructed from the 'Brahms' digital hologram. 


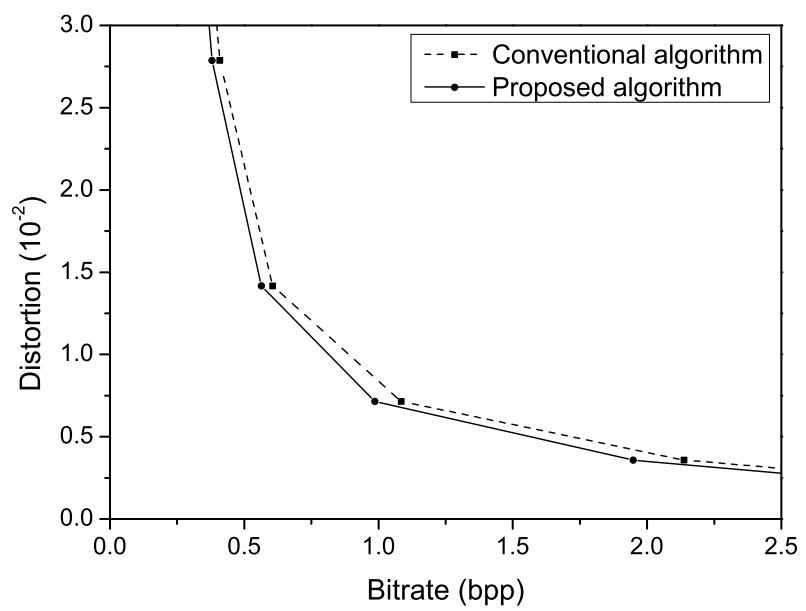

Fig. 7. Rate and distortion curve of the proposed algorithm compared with the conventional method. The rate means the average bits per pixel to encode the imaginary part object image reconstructed from the 'Dice' digital hologram.

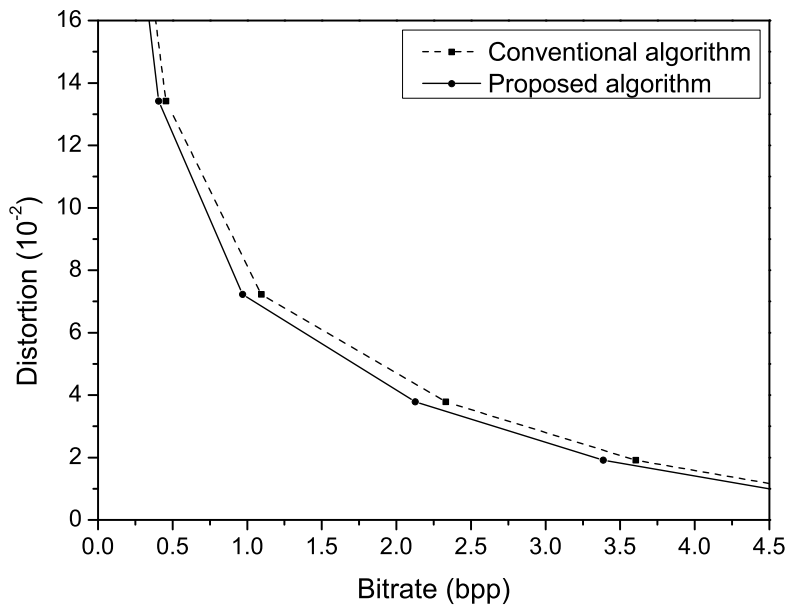

Fig. 8. Rate and distortion curve of the proposed algorithm compared with the conventional method. The rate means the average bits per pixel to encode the imaginary part object image reconstructed from the 'Copper screw' digital hologram. 


\section{Conclusion}

In this paper, we proposed an adaptive compression algorithm for digital holograms using the correlation in the reconstructed images. The object image reconstructed from a digital hologram is inherently a complex wave field, and thus composed of the real and imaginary parts. The real and imaginary part images are wavelet trasnformed and the transform coefficients are progressively encoded using the SPIHT method. The two images yield a relatively strong correlation each other, which can be exploited to improve the compression performance for digital holograms. Specifically, the result of significance coding for the real part image is employed as contexts of arithmetic coder to encode the imaginary part image. Experimental results showed that the proposed context-adaptive compression algorithm for digital holograms improved the coding gain compared with the conventional state-of-the-art method.

Acknowledgments. This research was supported by Basic Science Research Program through the NRF of Korea funded by the Ministry of Education, Science and Technology (2010-0006595).

\section{References}

1. http://www.edge.no/projects/holovision/

2. Antonini, M., Barlaud, M., Mathieu, P., Daubechies, I.: Image coding using wavelet transform. IEEE Trans. Image Processing 1(2), 205-220 (1992)

3. Darakis, E., Naughton, T.J., Soraghan, J.J.: Compression defects in different reconstructions from phase-shifting digital holographic data. Appl. Opt. 46(21), 45794586 (2007)

4. Darakis, E., Soraghan, J.J.: Use of Fresnelets for phase-shifting digital hologram compression. IEEE Trans. Image Processing 15(12), 3804-3811 (2006)

5. Darakis, E., Soraghan, J.J.: Reconstruction domain compression of phase-shifting digital holograms. Appl. Opt. 46(3), 351-356 (2007)

6. Frauel, Y., Naughton, T.J., Matoba, O., Tajahuerce, E., Javidi, B.: Three-dimensional imaging and processing using computational holographic imaging. Proc. of IEEE 94(3), 636-653 (2006)

7. Gabor, D.: A new microscopic principle. Nature 161, 777-778 (1948)

8. Goodman, J.W., Lawrence, R.W.: Digital image formation from electronically detected holograms. Appl. Phys. Lett. 11, 77-79 (1967)

9. Moffat, A., Neal, R., Witten, I.H.: Arithmetic coding revisited. In: Proc. IEEE Data Compression Conference, pp. 202-211 (March 1995)

10. Naughton, T.J., Frauel, Y., Javidi, B., Tajahuerce, E.: Compression of digital holograms for three-dimensional object reconstruction and recognition. Appl. Opt. 41, 4124-4132 (2002)

11. Said, A., Pearlman, W.: A new, fast, and efficient image codec based on set partitioning in hierarchical trees. IEEE Trans. Circuits Syst. Video Technol. 6, 243-250 (1996)

12. Schnars, U., Jueptner, W.: Digital holography: digital hologram recording, numerical reconstruction, and related techniques. Springer, Heidelberg (2005) 
13. Schnars, U., Jüptner, W.: Direct recording of holograms by a CCD target and numerical reconstruction. Appl. Opt. 33, 179-181 (1994)

14. Schnars, U., Jüptner, W.: Digital recording and numerical reconstruction of holograms. Meas. Sci. Technol. 13, R81-R101 (2002)

15. Shortt, A.E., Naughton, T.J., Javidi, B.: Compression of digital holograms of threedimensional objects using wavelets. Opt. Expr. 14(7), 2625-2630 (2006)

16. Shortt, A.E., Naughton, T.J., Javidi, B.: Histogram approaches for lossy compression of digital holograms of three-dimensional objects. IEEE Trans. Image Processing 16(6), 1548-1556 (2007)

17. Yamaguchi, I., Zhang, T.: Phase-shifting digital holography. Opt. Lett. 13(9), 12681270 (1997) 